\title{
Dual-energy Computed Tomography Scan: A New Imaging Option
}

\author{
Rudrajit Paul
}

\begin{abstract}
Dual-energy computed tomography scan (DECT) is emerging as a promising imaging technique with diverse applications. It is becoming useful in many fields like rheumatology, nephrology, and oncology. This article gives a brief description of the current and future applications of DECT.
\end{abstract}

Keywords: Angiography, Computed tomography scan, Crystal arthropathy, Oncology, Renal calculi.

How to cite this article: Paul R. Dual-energy Computed Tomography Scan: A New Imaging Option. Bengal Physician Journal, September-December 2018;5(3):36-39

\section{Source of support: Nil}

Conflict of interest: None

\section{INTRODUCTION}

Computed tomographic (CT) scan is an indispensable part of modern medical practice. Diverse specialties, including neuromedicine, orthopedics, radiotherapy and rheumatology use the CT scan in various forms for daily clinical management. With the advances in technology, the scope of CT scan has also expanded rapidly. A recent development in CT scan technology is the DECT, also called spectral CT scan. ${ }^{1}$ The basic principles for DECT were conceptualized almost 30 years ago, but the practical applications have become possible only in recent times. DECT was first introduced in 2005-2006 but it has seen remarkable development in the last decade. However, many aspects of the DECT imaging still need validation and DECT usage is still restricted mainly to academic institutions.

\section{Physics Behind the Application}

The CT scan is a modified form of X-ray imaging. Conventional CT scan, as is used widely, depends on X-ray beams generated from a single source, which are captured

\footnotetext{
Associate Professor

Department of Critical Care Medicine, Postgraduate Medical Education and Research and Seth Sukhlal Karnani Memorial Hospital, Kolkata, West Bengal, India

Corresponding Author: Rudrajit Paul, Associate Professor, Department of Critical Care Medicine, Postgraduate Medical Education and Research and Seth Sukhlal Karnani Memorial Hospital, Kolkata, West Bengal, India, e-mail: r.paul. medicalcollege@gmail.com
}

by one or more detectors. ${ }^{1}$ The resultant image depends on the attenuation of $X$-ray beams by various tissues and organs. But in many cases, two tissues in proximity may have almost the same attenuation (e.g., calcified plaques and blood with iodine contrast) which makes their differentiation difficult. ${ }^{2}$ The conventional Hounsfield unit numbers ascribed to various tissues in CT scan are often arbitrary and there is considerable overlap of Hounsfield unit numbers between various tissues. This gives rise to ambiguity in interpretation. In DECT, there are two sources of X-ray beams (typically 80 and $140 \mathrm{kVp}$ ). ${ }^{1}$ This helps to generate additional information about the tissues being analyzed. This may help in the differentiation of tissues which look almost the same in single energy CT scan. In DECT, besides Hounsfield units, CT number ratio is used to differentiate various tissues.

In DECT, the two X-ray sources and their detectors are placed orthogonal to each other. The two sets of data are then digitally reconstructed to generate the single final image. $^{2}$ The software is used to give various color coding to the tissues. Another technical variation of this imaging modality is using a single high energy $\mathrm{X}$-ray source and two types of detectors: one for low-energy data and one for high energy. ${ }^{2}$ This is called the multilayer detector technology and this is found to be comparable to the dual energy source variety. ${ }^{2}$

\section{Multiple Detector Computed Tomography}

A similar technology which is also becoming popular simultaneously is multi-detector CT (MDCT) scan. Here, there are multiple rows of detectors. This is also called a multislice CT scan. But the source of energy is single. True 3D reconstruction of organs and vessels is possible with MDCT. Also, imaging time is reduced greatly.

\section{Clinical Applications}

The full potential of DECT is still being studied but already quite a few important applications have been documented. The radiation dose of DECT is comparable to or even, less than conventional CT imaging.

\section{Computed Tomography Angiography}

One of the most helpful applications of DECT is in the field of CT angiography. The dual energy algorithm may be used 
to remove bone images (calcium subtraction technique), thereby making the iodinated contrast in blood vessels starkly prominent. ${ }^{2}$ Studies in cerebral angiography have shown that DECT is comparable to the current gold standard, digital subtraction angiography (DSA). ${ }^{3}$ Especially, vascular lesions like aneurysms near the base of the skull are often difficult to detect in conventional CT scan due to bone artefact. ${ }^{3}$ In such cases, DECT may be of immense use. Moreover, in DECT, radiation exposure is significantly reduced compared to DSA. ${ }^{3}$ In peripheral arterial angiography too, DECT has been shown to be effective. ${ }^{4}$

\section{Blood Pool Imaging}

This is the method of imaging whereby the perfused blood volume can be measured. ${ }^{2}$ This can help in the diagnosis of conditions with decreased regional blood flow, like pulmonary embolism (PE). ${ }^{2}$ In the diagnosis of PE, DECT or multi-detector CT scan (MDCT) has overtaken ventilation-perfusion scan or pulmonary angiography as the imaging method of choice. ${ }^{5}$ However, the MDCT is limited in its ability to detect perfusion defects at the subsegmental pulmonary artery level. ${ }^{5}$ DECT is much better in this respect and can detect perfusion defects in vessels as small as $3 \mathrm{~mm}$ in diameter. ${ }^{5}$ The sensitivity and specificity of DECT in detecting PE has been shown to be close to $100 \%$ in some studies. ${ }^{6}$

Dual-energy computed tomography (DECT) has also shown to be effective in detecting areas of poor myocardial perfusion. ${ }^{7}$ In some studies, the results are comparable to cardiac SPECT and MRI. ${ }^{7}$ Stress perfusion DECT is said to have a complementary role to coronary angiography in diagnosing hemodynamically significant stenosis. DECT can be done with any heart rate; in contrast, conventional CT of the heart requires a stable heart rate at 60-70 beats / min. However, cardiac uses of DECT are still experimental.

\section{Renal Stones}

A DECT can help in urinary stone characterization. Not only the calculus is diagnosed, but its approximate composition can also be determined from the images. ${ }^{2}$ The major differentiation is between uric acid and nonuric acid stones. Uric acid stones may be detected with almost $100 \%$ sensitivity. ${ }^{8}$ For other types, the sensitivity is lower, but still, the composition of non-uric acid stones (like cysteine or oxalate) can also be determined in many cases by DECT. ${ }^{2}$ A 2013 study by Liu et al. have shown that the accuracy of DECT in differentiating oxalate and hydroxyapatite urinary stones was comparable to urine analysis. ${ }^{9}$ Recently commercial software is available that can color code the stones with various chemical composition, thereby making interpretation of images much easier, even for the non-radiologist.

\section{Joint Imaging}

One major use of DECT in rheumatology is the diagnosis of crystal arthropathies. ${ }^{2}$ DECT images can differentiate uric acid from calcium salts easily. The gold standard for diagnosis of crystal arthropathies still remains joint fluid study under a polarising microscope. ${ }^{10}$ But a recent metaanalysis has shown that DECT has a pooled sensitivity of $87 \%$ and specificity of $84 \%$ in diagnosing gout. ${ }^{10}$ Thus, this can be a valuable non-invasive tool. However, joint imaging by DECT is susceptible to artifacts and false positive results. Although DECT is the best for diagnosis of chronic gout, it is also sometimes useful in acute conditions. ${ }^{11}$ Thus, this can be a valuable tool for diagnosis in cases of acute arthropathy, especially in cases where joint fluid aspiration in technically difficult.

A DECT is also useful in the diagnosis of pseudogout. ${ }^{12}$ It is especially helpful for joints where aspiration of joint fluid is difficult, like an atlantoaxial joint or interphalangeal joint. $^{12}$

\section{Atherosclerotic Plaques}

A DECT is a valuable tool in diagnosing atherosclerotic plaques in large or small vessels and assessing the patency of vessels. ${ }^{2}$ One major advantage of DECT is its ability to detect the composition of the plaques, thereby performing a "virtual histology". ${ }^{13}$ This can help in determining the prognosis of a plaque by assessing its potential for rupture.

\section{Other Applications}

- Oncology: In oncology, DECT can be used to characterize tissues, thereby differentiating tumor mass from surrounding benign tissues and avoiding radiation to normal tissues. Also, tumor recurrence can be detected. ${ }^{14}$ In some cases, DECT images have been found to be comparable to even FDG-PET scan in detecting malignant lesions. ${ }^{15}$ Another advantage of DECT, especially in gynecological cancers, is that it can detect the total tumor volume, thereby helping in prognostication. ${ }^{15}$ In the future, even the tumor tissue characterization may be possible with DECT, thereby performing virtual histology. ${ }^{15}$

- Virtual noncalcium images: In DECT images, the calcium signals from bones can be removed by software and this can be used to detect bone marrow edema. ${ }^{2}$ This can be a valuable tool in the imaging of injuries or arthritis. In a recent study, DECT has been found to be comparable to MRI in the setting of fractures. ${ }^{16}$ This is a significant advancement because often, in trauma patients, MRI is difficult to perform due to difficulty in positioning and the long time of image acquisition. Now, whether DECT may be useful 
in sacroiliitis is still a matter of research. But a recent case report has shown that DECT can be used successfully in the diagnosis of gouty sacroiliitis. ${ }^{17}$ However, the application of DECT for spondyloarthropathy is still debatable.

\section{Silicone Breast Implant}

DECT can be used to detect silicone leak from breast implants. ${ }^{2}$ A small amount of silicone leak from the implants can have almost the same attenuation as surrounding soft tissues and can be difficult to differentiate in conventional CT scan. But DECT can detect this leak easily. MRI is the imaging technique of choice for assessing the integrity of breast implants. ${ }^{18}$ But it cannot always detect lymph node spread of the silicone, and moreover, MRI is not always covered by insurance. ${ }^{18} \mathrm{DECT}$ can detect rupture of the implant, the extent of soft tissue spread and lymph node involvement: all in one image acquisition time. ${ }^{18}$

\section{Renal or Liver Mass Characterization}

Sometimes a renal or hepatic mass can be a diagnostic puzzle after conventional CT scan. Very small lesions are often not characterized properly in a CT scan, and the patient may need further invasive procedures for diagnosis. DECT can help in these cases by the iodine images; small cysts and metastases can be differentiated easily. ${ }^{1}$ Malignant lesions (primary or secondary) usually show iodine uptake. In the kidney, hyperattenuating cysts can be differentiated from malignant masses. ${ }^{19}$ In the near future, DECT may also be used to quantify the degree of hepatic steatosis or fibrosis. ${ }^{20}$ Also, it is possible to differentiate hemangioma, hepatocellular carcinoma, metastases, and bile duct cysts in the liver by DECT. ${ }^{20}$

Similarly, iso attenuating masses like adenocarcinoma or pancreas may be better detected with DECT. ${ }^{20}$ Both hypoand hypervascular lesions can be detected with DECT. This can save unnecessary biopsy and other interventions for diagnosis.

\section{Shortcomings and Future Applications}

Artifacts in image acquisition is a common problem in DECT. This can compromise the quality of the images. However, newer more advanced software is being developed to reduce such aberrations to a minimum.

In the future, multi-energy CT scans may also become a reality. That will reduce imaging time even further. In the future, it may be possible to use two different contrasts with different dual-energy indices. This may lead to better image quality for procedures like CT enterography.

\section{Take Home Messages}

- DECT scan is revolutionizing tissue imaging, especially for detecting the composition of renal stones and joint crystals

- This is possible at comparable or even reduced radiation doses, compared to conventional CT

- In many cases, like bone marrow edema imaging or myocardial perfusion assessment, it may be equivalent to MRI or SPECT scans

- In oncology, it may help in prognostication of diseases.

\section{REFERENCES}

1. Grajo JR, Patino M, Prochowski A, et al. Dual energy CT in practice: Basic principles and applications. Appl Radiol. 2016;45:6-12.

2. McCollough $\mathrm{CH}$, Leng S, Yu L, et al. Dual- and Multi-Energy CT: Principles, Technical Approaches, and Clinical Applications. Radiology 2015; 276: 637-653.

3. Zhang L, Wu S, Niu J, et al. Dual-Energy CT Angiography in the Evaluation of Intracranial Aneurysms: Image Quality, Radiation Dose, and Comparison With 3D Rotational Digital Subtraction Angiography. AJR 2010; 194:23-30.

4. Almutairi A, Sun Z, Poovathumkadavi A, et al. Dual Energy CT Angiography of Peripheral Arterial Disease: Feasibility of Using Lower Contrast Medium Volume. PLoS One. 2015; 10:e0139275.

5. Lu GM, Wu SY, Yeh BM, et al. Dual-energy computed tomography in pulmonary embolism. Br J Radiol. 2010; 83: 707-718

6. Fink C, Johnson TR, Michaely HJ, et al. Dual-energy CT angiography of the lung in patients with suspected pulmonary embolism: initial results. Rofo 2008;180:879-883.

7. Jin KN, De Cecco CN, Caruso D, et al. Myocardial perfusion imaging with dual energy CT. Eur J Radiol. 2016;85:19141921.

8. Rompsaithong U, Jongjitaree K, Korpraphong P, et al. Characterization of renal stone composition by using fast kilovoltage switching dual-energy computed tomography compared to laboratory stone analysis: a pilot study. AbdomRadiol (NY). 2018 Sep 26.

9. YLiu Y, Qu M, Carter RE, Leng S, et al. Differentiating Calcium Oxalate and Hydroxyapatite Stones In Vivo Using Dual-Energy CT and Urine Supersaturation and $\mathrm{pH}$ Values. AcadRadiol. 2013; 20: 1521-1525

10. Ogdie A, Taylor WJ, Weatherall M, et al. Imaging modalities for the classification of gout: Systematic literature review and meta-analysis. Ann Rheum Dis 2015; 74: 1868-1874.

11. Nicolaou S, Yong-Hing CJ, Galea-Soler S, Hou DJ, Louis L, Munk P. Dual-energy CT as a potential new diagnostic tool in the management of gout in the acute setting. Am J Roentgenol 2010; 194: 1072-1078.

12. Tanikawa H, Ogawa R, Okuma K, et al. Detection of calcium pyrophosphate dihydrate crystals in knee meniscus by dual-energy computed tomography. Journal of Orthopaedic Surgery and Research2018;13:73.

13. Obaid DR, Calvert PA, Gopalan D, et al. Dual-energy computed tomography imaging to determine atherosclerotic plaque composition: A prospective study with tissue validation. J CardiovascComputTomogr. 2014;8:230-237. 
14. Simons D, Kachelriess M, Schlemmer HP. Recent developments of dual-energy CT in oncology. Eur Radiol. 2014;24:930-939.

15. Benveniste AP, Faria SC, et al. Potential Application of DualEnergy CT in Gynecologic Cancer: Initial Experience. American Journal of Roentgenology. 2017;208:695-705.

16. Ali IT, Wong WD, Liang T, et al. Clinical Utility of DualEnergy CT Analysis of Bone Marrow Edema in Acute Wrist Fractures. AJR Am J Roentgenol. 2018;210:842-847.

17. Ao T, Shoda H, Yamamoto K. Two Cases of Gouty Sacroiliitis Evaluated by Dual-energy Computed Tomography. The Journal of Rheumatology 2016;43:1146-1147.
18. Glazebrook KN, Leng S, Jacobson SR, et al. Dual-Energy CT for Evaluation of Intra- and Extracapsular Silicone Implant Rupture. Case Reports in Radiology 2016;2016.

19. Mileto A, Allen BC, Pietryga JA, et al. Characterization of Incidental Renal Mass With Dual-Energy CT: Diagnostic Accuracy of Effective Atomic Number Maps for Discriminating Nonenhancing Cysts From Enhancing Masses. American Journal of Roentgenology. 2017;209:W221-230.

20. Lestra T, Mule S, Millet I, et al. Applications of dual energy computed tomography in abdominal imaging. Diagnostic and Interventional Imaging. 2016;97:593-603. 